\title{
A New Approach of Hetero-Cladding for Design of Compact Si Photonic Directional Coupler
}

This paper was downloaded from TechRxiv (https://www.techrxiv.org).

\section{LICENSE}

CC BY 4.0

SUBMISSION DATE / POSTED DATE

06-10-2021/12-10-2021

\section{CITATION}

MISHRA, MADHUSUDAN; Das, Nikhil (2021): A New Approach of Hetero-Cladding for Design of Compact Si Photonic Directional Coupler. TechRxiv. Preprint. https://doi.org/10.36227/techrxiv.16750399.v1

$\mathrm{DOI}$

10.36227/techrxiv.16750399.v1 


\title{
A New Approach of Hetero-Cladding for Design of Compact Si Photonic Directional Coupler
}

\author{
Madhusudan Mishra, Student Member, IEEE, and Nikhil Ranjan Das, Sr. Member, IEEE
}

\begin{abstract}
In this letter, we propose a new approach of hetero-cladding for realization of compact CMOS compatible silicon photonic directional couplers. The proposed hetero-cladding comprises ferroelectric $\mathrm{BaTiO}_{3}$ (BTO) and $\mathrm{SiO}_{2}$ to control the evanescent mode within the structure. The results show very small and identical coupling length for both TE and TM modes with reduced device cross-section, which promises for a huge reduction in the footprint of both conventional and programmable photonic integrated circuits. The concept can also be utilized to design compact, low loss and energy efficient phase shifters, other types of couplers, sensors etc.
\end{abstract}

Index Terms-Photonic Integrated Circuit, Reduced footprint, Programmable PIC, Directional coupler.

\section{Introduction}

$\mathrm{C}_{\mathrm{i}}$ MOS compatibility is an important driving force for increased interest in silicon based integrated photonic devices [1-4]. This, along with useful properties of light lead to numerous applications of cutting edge Si photonic technology [5-10]. However, large size of photonic devices compared to the state-of-the-art electronic counterparts leads to larger footprint of Si photonic integrated circuits (PICs). This severely limits the number of on-chip device integration, hence leads to less functionality of the PICs. To avoid it up to a certain extent, recently, programmable PICs have been developed to facilitate post-fabrication manipulation and on-demand reconfiguration of PICs with variety of functionalities [11-13]. These employ two-dimensional meshes of Mach-Zehnder (MZ) couplers as their fundamental building elements with a square, triangular, or hexagonal pattern as their unit cell $[11,12,14]$. However, since the size of currently used on-chip conventional MZ couplers is large (few 10's of $\mu \mathrm{m}$ ), their assembly in a huge number again contributes to extremely large footprint of programmable PICs. To reduce the footprint and making those energy efficient, cost effective and multi-functional, it is very essential to design compact MZ couplers.

In search of ultra-compact couplers, researchers have made use of plasmonics, photonic crystals and other techniques [1521]. However, most of those follow unconventional design rules with abundant structural modifications. Realization of

Submitted on October 6, 2021. This work was financially supported by Department of Science and Technology (DST), Govt. of India, through INSPIRE Fellowship program under Grant IF150280.

Madhusudan Mishra is with the Institute of Radio Physics and Electronics, 92, A.P.C. Road, University of Calcutta, Kolkata 700009, India (e-mail: madhusudandbt@gmail.com) ultra-compact MZ couplers without manipulating the cores of regular on-chip conventional bus waveguides is still a major concern. Using ferroelectric BTO as cladding material has been shown to be useful in reducing length of the switching device. BTO is being widely used in Si photonics due to its low loss, high quality growth on Si and its very high Pockels coefficient $(\sim 1000 \mathrm{pm} / \mathrm{V})$ etc. [22-26]. It's refractive index $(n)$ varies from $2.41\left(n_{o}\right)$ to $2.36\left(n_{\mathrm{e}}\right)$ tracing the orientation of its ferroelectric domains from in-plane ( $a$-axis) to out-of-plane ( $c$-axis) with application of suitable amount of electric field $[27,28]$. This huge modulation (i.e., $\Delta n=0.05$ ) is fast (takes few tens of microseconds) and quite non-volatile in nature [29-31]. In the proposed approach to tailor only the cladding material, BTO is used as one of the materials for the hetero-cladding, the other being $\mathrm{SiO}_{2}$. This creates an asymmetry in the dielectric mirror around the cores, which enhances the coupling process by allowing more light in the coupling region.

\section{Schematic Structure With Proposed Design}

The schematic of the device structure with a new approach to design using $\mathrm{SiO}_{2}-\mathrm{BTO}-\mathrm{SiO}_{2}$ hetero-cladding of thickness ' $t$ ' is shown in Fig. 1(a). The coupling gap is taken as $200 \mathrm{~nm}$ to ensure it doesn't violate the minimum experimental limit. In this concept of hetero-cladding, our aim is to gather more light in the cladding which take part in coupling and reducing the light spreading in other cladding region. At the same time, the hetero-cladding also includes the technique of releasing more light from core to cladding without modifying the core structure unlike the slotted/trenched core structure [32]. The whole cladding region of the hetero-cladded coupler is hence devided into two parts, named "Region-1" and "Region-2" (indicated in figure). Region-1 contributes for light guiding, while region-2 is involved in coupling process. Fig. 1(b) shows the schematic of the refractive index profile of the given structure. The idea here is to keep region-1 with a cladding material of high refractive index contrast (w.r.t core) and to replace region-2 with a low refractive index contrast material, to help the mode evanescent gather there to enhance the coupling as shown later. Moreover, the two additional dielectric interfaces formed at the junction of both the claddings (indicated as dashed orange lines in Fig. 1(b)), will prevent spreading of the mode evanescent in

Nikhil Ranjan Das is with the Institute of Radio Physics and Electronics, 92, A.P.C. Road, University of Calcutta, Kolkata 700009, India (e-mail: nrd@ieee.org). 


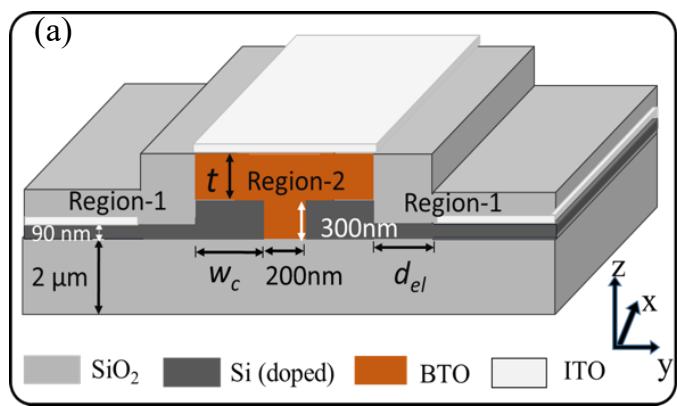

region-1. As it is shown in Fig. 1(a), BTO is used as cladding for region-2, by keeping region-1 as $\mathrm{SiO}_{2}$. This kind of cladding design creates a strong reflection of light at the outer walls of the cores (red lines in Fig. 1(b)), whereas a weak reflection at the inner wall and top of the cores (solid orange lines in Fig. 1(b)). This asymmetry in reflection helps pushing the mode shape more towards the BTO cladding i.e. to region2, without seeking for any modification in the core. Weak reflection at inner side wall and top of the cores could also help the device become less sensitive to roughness at the corecoupling region interface.

The proposed structure can be fabricated using the procedures indicated in literature [26, 29, 33-35]. For example, after the growth (/patterning) of the doped Si-core waveguide, BTO layer can be deposited around the Si-cores using pulsed layer deposition technique [29]. A mask can be used along with a photoresist to etch out the BTO from the sides using inductively coupled plasma (ICP) etching technique leaving BTO cladding only on the top [35]. It may be mentioned here that a slight misalignment of the BTO edge and Si-core edge won't affect the performance reported in this study. The side cladding now can be filled with growth of $\mathrm{SiO}_{2}$.

To exploit the electro-optic effect of ferroelectric BTO, three independent ITO electrodes could be employed (Fig. 1(a)) in two biasing configurations, say "biasing-1" (to set $n$ to $n_{e}$ ) and "biasing-2" (to set $n$ to $n_{o}$ ) (Fig. 1(c)) as reported earlier [28,36]. Initially $n$ is taken as $n_{o}$. Also, both the cores are n-doped $\left(\sim 10^{18}\right)$ to behave as a part of bottom ITO electrodes.

\section{Numerical Results and SElection of Structure PARAMETERS}

This part of study employs FEM based simulator (COMSOL) to optimize the structure parameters as follows.

\section{A. Estimation of Core Width and Coupling Length}

Fig. 2 shows a comparison between the mode shapes of the proposed hetero-cladding and the uniform (BTO) cladding structure. Comparing Figs. 2(a) and (b) it can be seen that, the presence of TM mode evanescent in the central BTO cladding
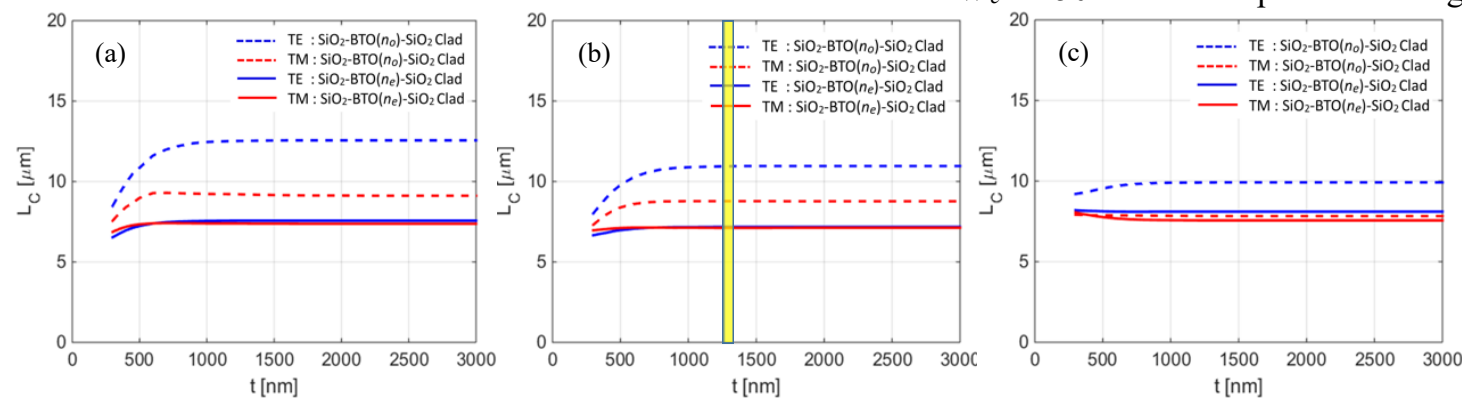

Fig. 3. Coupling length of directional coupler with hetero-cladding for both $n=$ $n_{o}$ (dashed line) and $n=n$, (solid line) for wave guide widths of (a) $400 \mathrm{~nm}$, (b) $450 \mathrm{~nm}$, (c) $500 \mathrm{~nm}$. 


\section{B. Estimation of Transverse Dimension}

As shown in Fig.2, light in cladding is mainly present in region-2, with its negligible presence in region-1. This greatly reduces the transverse dimension and power loss of the device. Absence of mode evanescent beyond few 100's of nanometers from outside wall of the cores allows the side electrodes sit closer to them without any significant contribution to insertion loss. This leads to the need of less applied voltage.

The amount of loss experienced by the structure (with optimized core-to-side electrode gap $\left(d_{e l}\right)$ of $500 \mathrm{~nm}$ and thickness $t$ of $1300 \mathrm{~nm}$ ) for TE and TM modes are around 0.052 $\mathrm{dB}$ and $0.057 \mathrm{~dB}$ respectively with $n=n_{o}$, and is $0.035 \mathrm{~dB}$ and $0.040 \mathrm{~dB}$ for $n=n_{e}$. The estimated loss for uniform BTO cladding case for same structure parameters are around 0.135 $\mathrm{dB}$ and $0.179 \mathrm{~dB}$ respectively for TE and TM modes with $n=$ $n_{o}$, and is $0.138 \mathrm{~dB}$ and $0.14 \mathrm{~dB}$ with $n=n_{e}$; thus showing 3-4 times improvement in the proposed hetero-cladding structure.

Finally, the optimized parameter values are summarized in Table I. It can be seen that, the values of minimum $L_{c}$ may be kept $\sim 7.1 \mu \mathrm{m}$ for both TE and TM modes, which may further reduce if coupling assisted by bending is considered as in an actual design, discussed in the following sub-section.

TABLE I

OPTIMIZED PARAMETER VALUES OF THE PROPOSED STRUCTURE

\begin{tabular}{lll}
\hline \hline \multirow{2}{*}{ Symbol } & \multicolumn{1}{c}{ Structure Parameters } & Optimized Values \\
\hline$t$ & Thickness of cladding & $1300 \mathrm{~nm}$ \\
$d_{e l}$ & Core to side electrode Spacing, & $500 \mathrm{~nm}$ \\
$W_{c}$ & Width of core & $450 \mathrm{~nm}$ \\
$L_{c}$ & Minimum coupling length for TE & $11 \mu \mathrm{m}(\mathrm{TE})$ \\
& and TM mode for $n=n_{o}$ & $9 \mu \mathrm{m}(\mathrm{TM})$ \\
$L_{c}$ & Minimum coupling length for TE & $7.18 \mu \mathrm{m}(\mathrm{TE})$ \\
& and TM mode for $n=n_{e}$ & $7.12 \mu \mathrm{m}(\mathrm{TM})$ \\
& & \\
\hline \hline
\end{tabular}

\section{Optical Design}

Using suitable dimensions as discussed above, the schematic of optical design and the power coupling plot are shown in Fig.4. As shown in Fig. 4(a), the structure uses a bending radios $\left(b_{r}\right)$ of $8 \mu \mathrm{m}$ with hetero cladding only in the straight coupling region. Fig. 4(b) shows the light coupling (seen to be $\sim 97 \%$ from simulation) with an insertion loss of about $0.14 \mathrm{~dB}$. Thus, high power coupling can be achieved with this design for reduced footprint. This $L_{c}$ is less than the minimum $L_{c}$ mentioned in Table I because of the bending arms. Fig. 4 (c) shows power coupling assuming $50 \mathrm{~nm}$ of under-filling at the bottom of coupling gap (assuming any fabrication constraint).

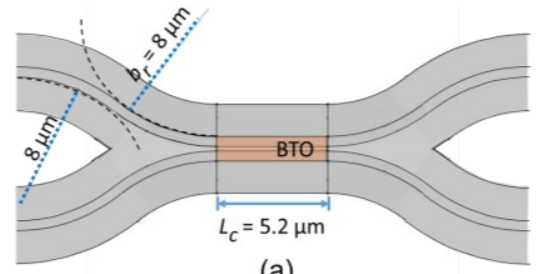

(a)

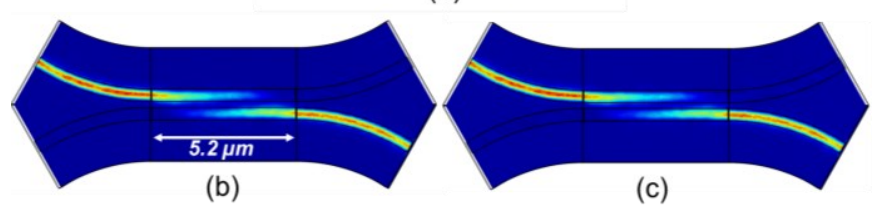

Fig. 4. (a) Schematic of coupler (b) power coupling for $n=n_{e}$ without under filling and (c) with $50 \mathrm{~nm}$ of under filling.
Simulations show that the power coupling here is $2 \%$ less. It may be mentioned here that the power coupling can be tuned to some extent by applying a suitable bias as reported earlier [41].

\section{CONCLUSION}

A new approach of hetero-cladding $\left(\mathrm{BTO} / \mathrm{SiO}_{2}\right)$ has been proposed for realization of compact silicon photonic devices through realization of compact directional coupler. The design is capable to make the coupler more than $60 \%$ smaller compared to traditional uniform $\mathrm{SiO}_{2}$ cladded structure. An optical design shows high power coupling using the designed small coupling length. The concept of hetero-cladding could also be used to improve the performance of other type of couplers, sensors, phase shifter, switches, power splitters etc.

\section{ACKNOWLEDGMENT}

The authors acknowledge Dr. Francesco Morichetti of Dipartimento di Electtronica, Informazione e Bioingegneria, Politecnico di Milano, Milan, Italy, for his useful advice and support on viability of design from experimental aspects. 


\section{REFERENCES}

[1] J. Xiao, Q. Wei, D. G. Yang, P. Zhang, N. He, G. Q. Zhang, T. L. Ren, X. P. Chen "A CMOS-Compatible Hybrid Plasmonic Slot Waveguide With Enhanced Field Confinement", IEEE Electron Device Letters 37, pp. 456458, April 2016. DOI: 10.1109/LED.2016.2531990

[2] M. Frounchi, G. N. Tzintzarov, A. Ildefonso, J. D. Cressler, "High Responsivity Ge Phototransistor in Commercial CMOS Si-Photonics Platform for Monolithic Optoelectronic Receivers", IEEE Electron Device Letters 42, pp. 196-199, Feb. 2021. DOI: 10.1109/LED.2020.3042941

[3] R. Won, "Integrating silicon photonics", Nature Photonics 4, pp. 498499, August 2010. DOI: https://doi.org/10.1038/nphoton.2010.189

[4] Y. Li, L. Yang, H. Zou, H. S. Zhang, X. H. Ma, Y. Hao, "Substrate Integrated Waveguide Structural Transmission Line and Filter on Silicon Carbide Substrate", IEEE Electron Device Letters 38, pp. 1290-1293, Sept. 2017. DOI: 10.1109/LED.2017.2734947

[5] D. Thomson, A. Zilkie, J. E. Bowers, T. Komljenovic, G. T. Reed1, L. Vivien, D. M. Morini, E. Cassan, L. Virot, J. M. Fédéli, J. M. Hartmann, J. H. Schmid, D. X. Xu, F. Boeuf, P. O'Brien, G. Z Mashanovich, M. Nedeljkovic "Roadmap on silicon photonics", Journal of Optics 18, pp.073003, June 2016, DOI: https://doi.org/10.1088/2040$\underline{8978 / 18 / 7 / 073003}$

[6] X. Wang, J. Liu, "Emerging technologies in Si active photonics", Journal of Semiconductors 39, June 2018. DOI:https://doi.org/10.1088/16744926/39/6/061001

[7] F. Giuseppe, D. Corte, S. Rao, "Use of Amorphous Silicon for Active Photonic Devices", IEEE Transactions on Electron Devices 60, pp.14951505, May 2013. DOI: 10.1109/TED.2013.2249069

[8] M. Chacinski, U. Westergren, B. Stoltz, L. Thylen , "Monolithically Integrated DFB-EA for $100 \mathrm{~Gb} / \mathrm{s}$ Ethernet", IEEE Electron Device Letters 29, pp. 1312-1314, Dec. 2008. DOI: 10.1109/LED.2008.2007222

[9] A. Noriki, K. Lee, J. Bea, T. Fukushima, T. Tanaka, M. Koyanagi, "Through-Silicon Photonic Via and Unidirectional Coupler for HighSpeed Data Transmission in Optoelectronic Three-Dimensional LSI", IEEE Electron Device Letters 33, pp. 221-223, Feb. 2012. DOI: 10.1109/LED.2011.2174608

[10] Z. Zhou, R. Chen, X. Li, T. Li, "Development trends in silicon photonics for data centers", Optical Fiber Technology 44, pp. 13-23, August 2018. DOI: https://doi.org/10.1016/j.yofte.2018.03.009

[11] W. Bogaerts, D. Pérez, J. Capmany, D. A. B. Miller, J. Poon, D. Englund, F. Morichetti, A. Melloni, "Programmable photonic circuits", Nature 586, pp. 207-216, October 2020. DOI: https://doi.org/10.1038/s41586-0202764-0

[12] D.P. López, "Principles, fundamentals, and applications of programmable integrated photonics", Adv. in Opt. and Photonics 12, pp. 709-785, 2020. DOI: https://doi.org/10.1364/AOP.387155

[13] H. Zhou, Y. Zhao, X. Wang, D. Gao, J. Dong, X. Zhang, "SelfConfiguring and Reconfigurable Silicon Photonic Signal Processor", ACS Photonics 7, pp. 792-799, February 2020. DOI: https://doi.org/10.1021/acsphotonics.9b01673

[14] D. Perez and I. Gasulla, "Toward programmable microwave photonics processors," J. Lightwave Technology 36, 519-532, 2018. DOI: 10.1109/JLT.2017.2778741

[15] C.-H. Du and Y. P. Chiou, "Vertical Directional Couplers with UltraShort Coupling Length Based on Hybrid Plasmonic Waveguides", Journal of Lightwave Technology 32, pp. 2065-2071, June 2014. DOI: 10.1109/JLT.2014.2318182

[16] L. Ye, Y. Zheng, J. Wang, H. Liao, R. Huang, "Miniature CMOS Stacked Spiral-Coupled Directional Coupler With -67-dB Isolation and - 0.8-dB Insertion Loss", IEEE Electron Device Letters 33, pp. 919-921, July 2012. DOI: 10.1109/LED.2012.2196749

[17] C. E. Garcia-Ortiz, V. Coello, E. Pisano, Y. Chen, S. I. Bozhevolnyi, "Plasmonic directional couplers using channel waveguides in random arrays of metal nanoparticles", Optics Express 27, pp. 22753-22763, 2019. DOI: https://doi.org/10.1364/OE.27.022753

[18] N. Janrao, and V. Janyani, "Ultra compact slow light photonic crystal directional coupler design with elliptical rods", Optik 124, pp. 3120-3124 2013. DOI: https://doi.org/10.1016/j.ijleo.2012.09.039

[19] H. Xie, J. Zheng, P. Xu, J. Yao, J. Whitehead, A. Majumdar, "UltraCompact Subwavelength-Grating-Assisted Polarization-Independent Directional Coupler", IEEE Photonics Technology Letters 31, pp. 15381541, August 2019. DOI: 10.1109/LPT.2019.2937890

[20] R. Haldar, V. Mishra, A. Dutt, S. K Varshney, "On-chip broadband ultracompact optical couplers and polarization splitters based on off-centered and non-symmetric slotted Si wire waveguides", J. Opt. 18, pp. 105801, September 2016. DOI: https://doi.org/10.1088/2040-8978/18/10/105801

[21] H. Sattari, A. Y. Takabayashi, Y. Zhang, P. Verheyen, W. Bogaerts, N. Quack "Compact broadband suspended silicon photonic directional coupler", Opt. Lett. 45, pp. 2997-3000, 2020. DOI: https://doi.org/10.1364/OL.394470

[22] F. Eltes, D. Caimi, F. Fallegger, M. Sousa, E. O'Connor, M. D. Rossell, B. Offrein, J. Fompeyrine, S. Abel, "Low-loss BaTiO3-Si waveguides for nonlinear integrated photonics", ACS Photonics 3(9), pp. 1698-1703, August 2016. DOI: https://doi.org/10.1021/acsphotonics.6b00350

[23] K. J. Kormondy, Y. Popoff, M. Sousa, F. Eltes, D. Caimi, M. D Rossell, M. Fiebig, P. Hoffmann, C. Marchiori, M. Reinke, M. Trassin, A. A Demkov, J. Fompeyrine, S. Abel, "Microstructure and ferroelectricity of $\mathrm{BaTiO}_{3}$ thin films on Si for integrated photonics," Nanotechnology 28, 075706, January 2017. DOI: https://doi.org/10.1088/1361-6528/aa53c2

[24] S. Abel, T. Stoferle, C. Marchiori, D. Caimi, L. Czornomaz, M. Stuckelberger, M. Sousa, B. J. Offrein, J. Fompeyrine, "A hybrid barium titanate-silicon photonics platform for ultraefficient electro-optic tuning," J. Lightwave Technol. 34, 1688-1693, December 2015. DOI: 10.1109/JLT.2015.2510282

[25] C. Xiong, W. H. P. Pernic, J. H. Ngai, J. W. Reiner, D. Kumah, F. J. Walker, C. H. Ahn, H. X. Tang, "Active Silicon Integrated Nanophotonics: Ferroelectric $\mathrm{BaTiO}_{3}$ Devices", Nano Lett. 1, pp. 14191425, January 2014. DOI: https://doi.org/10.1021/nl404513p

[26] E. L. Lin, A. B. Posadas, L. Zheng, J. E. Ortmann, S. Abel , J. Fompeyrine, K. Lai, A. A. Demkov, J. G. Ekerdt, "Atomic layer deposition of epitaxial ferroelectric barium titanate on $\mathrm{Si}(001)$ for electronic and photonic applications", J. Appl. Phys. 126, pp. 064101(1-9), August 2019. DOI: https://doi.org/10.1063/1.5087571

[27] M. J. Dicken, L. A. Sweatlock, D. Pacifici, H. J Lezec, K. Bhattacharya, H. A Atwater, "Electrooptic modulation in thin film barium titanate plasmonic interferometers," Nano Lett. 8, 4048-4052 November 2008. DOI: $10.1021 / \mathrm{n} 1802981 \mathrm{q}$

[28] M. Mishra, N. R. Das, A. Melloni, F. Morichetti, "Modelling domain switching of ferroelectric $\mathrm{BaTiO}_{3}$ integrated in silicon photonic waveguides," Opt. Commun. 448, 19-25, October 2019. DOI: https://doi.org/10.1016/j.optcom.2019.05.001

[29] I. M. Albo, S. Varotto, M. Asa, C. Rinaldi, M. Cantoni, R. Bertacco, F. Morichetti, "Non-volatile switching of polycrystalline barium titanate films integrated in silicon photonic waveguides," in Advanced Photonics 2018 (BGPP, IPR, NP, NOMA, Sensors, Networks, SPPCom, SOF), OSA Technical Digest (online) (Optical Society of America, 2018), paper ITu4I.2. DOI: https://doi.org/10.1364/IPRSN.2018.ITu4I.2

[30] T. Mikolajick, "The Past, the Present, and the Future of Ferroelectric Memories", IEEE Transactions on Electron Devices 67, NO. 4, pp.14341443, March 2020. DOI: 10.1109/TED.2020.2976148

[31] R. P. Shi, X. D. Huang, J. K. O. Sin, P. T. Lai "Y-Doped $\mathrm{BaTiO}_{3}$ as a Charge-Trapping Layer for Nonvolatile Memory Applications", IEEE Electron Device Letters 37, pp. 1555-1558, Dec. 2016. DOI: 10.1109/LED.2016.2615063

[32] M. Mishra and N. R. Das, "Trenched Core Waveguide Structure for Photonic Integrated Circuit", in "Computers and Devices for Communication", N. R. Das and Santu Sarkar (Eds.), Lecture Notes in Networks and Systems, Springer Nature Singapore Pte. Ltd. 147, 321 February 2021. DOI: 10.1007/978-981-15-8366-7 46

[33] Y.-J. Seo and W.-S. Lee, "Chemical Mechanical Polishing Characteristics of Barium Titanate Thin Films Using Mixed Abrasive Slurry", Journal of the Korean Physical Society 48, pp. 1651-1656, 2006. https://www.jkps.or.kr/journal/view.html?uid=7625\&vmd=Full

[34] Y. Li, C. Wang, Z. Yao, H. K. Kim, N. Y. Kim, "Comparative analysis of barium titanate thin films dry etching using inductively coupled plasmas by different fluorine-based mixture gas", Nanoscale Research Letters 9.1, September 2014. DOI: https://doi.org/10.1186/1556-276X-9$\underline{530}$

[35] C. Wang, H.K. Sung, N.Y. Kim, "Aerosol deposition-based micropatterning of barium titanate via sulphur hexafluoride inductively coupled plasma etching", Vacuum 114, pp. 49-53, April 2015. DOI: https://doi.org/10.1016/j.vacuum.2014.12.028

[36] M. Mishra, N. R. Das and F. Morichetti, "Waveguide design optimization for compact silicon photonic ferroelectric phase shifters", Applied Optics 59, pp. 4385-4391 May 2020. DOI: https://doi.org/10.1364/AO.390273 